\title{
Classification of the congruent embeddings of a tetrahedron into a triangular prism
}

\author{
H. Maehara and N. Tokushige
}

September 14, 2009

\begin{abstract}
Let $\mathbf{P}(t)$ denote an infinitely long right triangular prism whose base is an equilateral triangle of edge length $t$. Let $\mathcal{F}(t)$ be the family of those subsets of $\mathbf{P}(t)$ that are congruent to a regular tetrahedron of unit edge. We present complete classification of the members of $\mathcal{F}(t)$ modulo rigid motions within the prism $\mathbf{P}(t)$, for every $t>0$.
\end{abstract}

\section{Introduction}

Problems related to embedding or inscribing simplices into circular cylinders are considered by many authors, mostly to study the outer $j$-radii of simplices, or to compute the cylinders through the vertices of a simplex. See, e.g., Brandenberg, et al. [2, 3], Devillers, et al. [4], Pukhov [8], Schömer, et al. [9]. Maehara [7] treats embedding itself, and proved that all embeddings of a regular tetrahedron in a circular cylinder are equivalent modulo rigid motions within the cylinder.

In this paper, we classify the congruent embeddings of a regular tetrahedron in a right prism whose base is an equilateral triangle. This study arouse from the investigation [1] of the minimum size of an equilateral triangular hole in a plane through which a regular tetrahedron of unit edge can pass.

A regular tetrahedron with unit edge is simply called a unit tetrahedron. A right triangular prism $\mathbf{P}=\Delta \times \mathbb{R}$ with equilateral triangular base $\Delta$ is called simply a prism. The size of a prism $\mathbf{P}, \operatorname{size}(\mathbf{P})$, is the length of the edge of $\Delta$. A prism of size $t$ is denoted by $\mathbf{P}(t)$. An embedding of a unit tetrahedron in $\mathbf{P}$ means such a subset of $\mathbf{P}$ that is congruent to a unit tetrahedron. Two embeddings $T_{1}, T_{2} \subset \mathbf{P}$ of a unit tetrahedron in $\mathbf{P}$ are said to be equivalent (written as $T_{1} \sim T_{2}$ in $\mathbf{P})$ if it is possible to superpose $T_{1}$ on $T_{2}$ by a continuous rigid motion of $T_{1}$ within $\mathbf{P}$. More precisely, $T_{1} \sim T_{2}$ in $\mathbf{P}$ if there is a continuous map $F: T_{1} \times[0,1] \rightarrow \mathbf{P}$ such that

(1) for every $t \in[0,1]$, the map $f_{t}: T_{1} \rightarrow \mathbf{P}$ defined by $f_{t}(x)=F(x, t)$ gives an isometry from $T_{1}$ to $f_{t}\left(T_{1}\right)$, and 
(2) $f_{0}$ is the inclusion map, and $f_{1}\left(T_{1}\right)=T_{2}$.

The relation $\sim$ in $\mathbf{P}$ is clearly an equivalence relation. Let $v(t)$ denote the maximum number of mutually non-equivalent embeddings of $T$ in $\mathbf{P}(t)$. We prove the following.

\section{Theorem 1.1.}

$$
v(t)=\left\{\begin{array}{cl}
0 & \text { for } t<t_{0}:=\frac{1+\sqrt{2}}{\sqrt{6}} \\
6 & \text { for } t_{0} \leq t<t_{1}:=\frac{\sqrt{3}+3 \sqrt{2}}{6} \\
18 & \text { for } t_{1} \leq t<1 \\
1 & \text { for } 1 \leq t .
\end{array}\right.
$$

Thus, a unit tetrahedron can be embedded in $\mathbf{P}(t)$ if and only if $t \geq \frac{1+\sqrt{2}}{\sqrt{6}}$. This fact is used in [1] to prove that a unit tetrahedron can pass through an equilateral triangular hole in a plane if and only if the edge length of the triangular hole is at least $\frac{1+\sqrt{2}}{\sqrt{6}}$.

Let $v_{\circ}(t)$ denote the number of equivalence classes of the embeddings of a unit tetrahedron into an infinite circular cylinder of diameter $t$ modulo rigid motions within the cylinder. The number $v_{\circ}(t)$ is determined in [7]: $v_{\circ}(t)=0$ for $r<1$, and $v_{\circ}(t)=1$ for $r \geq 1$. Let $v_{\square}(t)$ be the number of equivalence classes of all embeddings of a unit tetrahedron into a square prism whose base is a square with diameter $t$, modulo rigid motions within the prism. Since a square of diagonal $t$ can be inscribed in a circle of diameter $t, v_{\circ}(t)=0$ for $r<1$ implies that $v_{\square}(t)=0$ for $t<1$, see also Itoh, et al. [5].

Problem. Determine $v_{\square}(t)$ for $t \geq 1$.

Throughout this paper, prisms are assumed to be vertically placed in $\mathbb{R}^{3}$, that is, their generators are parallel to the $z$-axis. Hence the intersection of a prism $\mathbf{P}$ and the $x y$-plane is an equilateral triangle.

\section{A cross embedding and a tangential embedding}

Lemma 2.1. Let $t_{0}=(1+\sqrt{2}) / \sqrt{6}$. Then $\mathbf{P}\left(t_{0}\right)$ contains a unit tetrahedron.

Proof. Put $h=t_{0} / 2=(1+\sqrt{2}) / \sqrt{24}$, and let $\Delta$ be the triangle on the $x y$-plane with vertices $( \pm h, 0,0),(0, \sqrt{3} h, 0)$. Then $\Delta$ is an equilateral triangle of edge length $t_{0}$, as easily verified. Put $k=(\sqrt{2}-1) / \sqrt{24}, \ell=1 / \sqrt{2}$, and define four points $A, B, C, D$ by

$$
A=(k, \ell,-h), B=(-h, 0,-k), C=(h, 0, k), D=(-k, \ell, h) .
$$

These four points span a unit tetrahedron, and their orthogonal projections on the $x y$-plane lie on $\triangle$, see Figure 1 . Thus the unit tetrahedron $A B C D$ is contained in $\mathbf{P}\left(t_{0}\right)=\Delta \times \mathbb{R}$. 


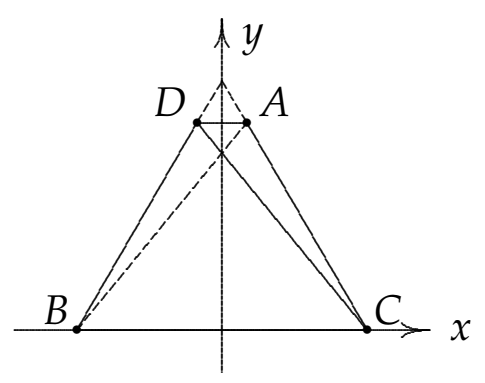

Figure 1: Top view of the tetrahedron in the triangular prism

This embedding is referred to as a cross embedding. Similarly, we can construct six different cross embeddings (modulo translations) by changing the face $\sigma$ of $\mathbf{P}\left(t_{0}\right)$ containing the edge $B C$, and by changing the crossing type $\epsilon$ (which can be done by changing the signs of the $z$-coordinates of $A, B, C, D$ ). These six different cross embeddings are denoted by

$$
\alpha(\sigma, \epsilon) \quad\left(\sigma=\sigma_{1}, \sigma_{2}, \sigma_{3}, \quad \epsilon=\epsilon_{1}, \epsilon_{2}\right),
$$

where $\sigma_{1}, \sigma_{2}, \sigma_{3}$ denotes the the faces of $\mathbf{P}\left(t_{0}\right)$, and $\epsilon_{1}=\rtimes, \epsilon_{2}=\chi$.

A tangential embedding $T \subset \mathbf{P}$ is an embedding such that some three vertices of $T$ lie on one and the same face of $\mathbf{P}$.

Lemma 2.2. Let $t_{1}:=(\sqrt{3}+3 \sqrt{2}) / 6 \approx 0.99578$. Then $\mathbf{P}\left(t_{1}\right)$ contains a tangential embedding of a unit tetrahedron.

Proof. Let $\Delta_{1}$ be the triangle on the $x y$-plane with vertices

$$
\bar{A}=\left(\frac{\sqrt{2}}{3}, 0,0\right), \bar{B}=\left(-\frac{\sqrt{3}+\sqrt{2}}{6}, 0,0\right), E=\left(-\frac{\sqrt{3}-\sqrt{2}}{12}, \frac{\sqrt{6}+1}{4}, 0\right) .
$$

A straightforward calculation shows that $\Delta_{1}$ is an equilateral triangle with edge length $t_{1}$. Let $T_{1}=A B C D$ be the tetrahedron with vertices

$$
\begin{aligned}
& A=\left(\frac{\sqrt{2}}{3}, 0, \frac{1}{3}\right), B=\left(-\frac{\sqrt{3}+\sqrt{2}}{6}, 0, \frac{\sqrt{6}-1}{6}\right), C=\left(\frac{\sqrt{3}-\sqrt{2}}{6}, 0,-\frac{\sqrt{6}+1}{6}\right), \\
& D=\left(0, \frac{\sqrt{6}}{3}, 0\right) .
\end{aligned}
$$

Figure 2 shows how the face $A B C$ is embedded in a face of $\mathbf{P}\left(t_{1}\right)$, see also Figure 5 in Section 4 . The vertex $D$ lies on another face of $\mathbf{P}\left(t_{1}\right)$. Then $T_{1}$ is a tangential embedding of $T$ in $\mathbf{P}\left(t_{1}\right)$.

Similarly, we can construct different tangential embeddings by changing the face $\sigma$ of $\mathbf{P}\left(t_{1}\right)$ that contains $A B C$, and changing the embedding type $\delta$ of $A B C$ in $\sigma$ in the following four different ways:
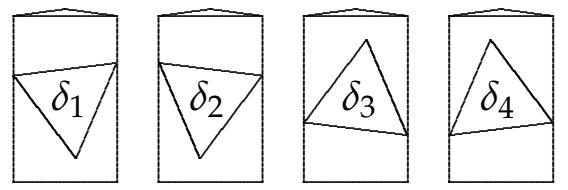


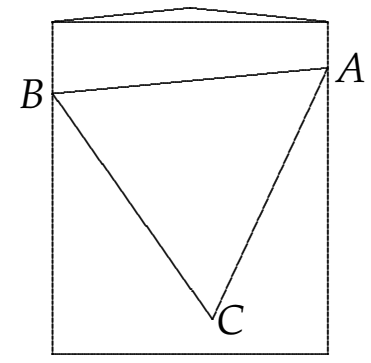

Figure 2: Face $A B C$ in a face of $\mathbf{P}\left(t_{1}\right)$

Thus, there are 12 different tangential embeddings modulo translations in $\mathbf{P}\left(t_{1}\right)$. They are denoted by

$$
\beta(\sigma, \delta) \quad\left(\sigma=\sigma_{1}, \sigma_{2}, \sigma_{3}, \quad \delta=\delta_{1}, \delta_{2}, \delta_{3}, \delta_{4}\right)
$$

From now on, we assume that the prisms $\mathbf{P}(t), t \in \mathbb{R}_{+}$are nested in such a way that they have the same center axis and parallel faces. Thus the sections of some two prisms by a horizontal plane look like $\triangle$. Then an embedding $T \subset \mathbf{P}(s)$ is naturally regarded as an embedding $T \subset \mathbf{P}(t)$ for $s<t$. Thus the embedding $\alpha(\sigma, \epsilon) \subset \mathbf{P}\left(t_{0}\right)$ is an embedding in $\mathbf{P}(t)$ for $t \geq t_{0}$, and $\beta(\sigma, \delta) \subset \mathbf{P}\left(t_{1}\right)$ is an embedding in $\mathbf{P}(t)$ for $t \geq t_{1}$.

\section{Conditions to reduce the containment size}

An interior vertex of $T \subset \mathbf{P}$ is a vertex of $T$ lying in the interior $\mathbf{P}^{\circ}$ of $\mathbf{P}$. A corner vertex of $T \subset \mathbf{P}$ is a vertex lying on the corner line of $\mathbf{P}$. For every point $P \in \mathbb{R}^{3}$, let $z(P)$ denote the $z$-coordinate of $P$, and $\bar{P}$ denote the orthogonal projection of $P$ on the $x y$-plane.

Lemma 3.1. Let $T \subset \mathbf{P}$ be an embedding. If

(1) Thas an interior vertex, or

(2) Thas at most one corner vertex,

then $T$ can be congruently moved into $\mathbf{P}^{\circ}$.

Proof. Let $T=A B C D$. A face of $\mathbf{P}$ that contains no vertex of $T$ is called an empty face. Note that if $\mathbf{P}$ has an empty face $\sigma$, then we can push $T$ slightly toward $\sigma$ so that $T$ goes into $\mathbf{P}^{\circ}$.

(1) First, note that if $T$ has two interior vertices, say, $A, B$, and $\mathbf{P}$ has no empty face, then $C, D$ must be corner vertices. In this case, a small rotation of $T$ around the line through the midpoint of $C D$ and perpendicular to the face containing $C D$ makes two faces of $\mathbf{P}$ empty. 
Now, suppose that $A$ is an interior vertex. If one of $B, C, D$, say, $D$, is not a corner vertex, then a small rotation of $T$ around the line $B C$ makes $A, D$ interior vertices. Suppose that $B, C, D$ are all corner vertices. Then no two of them lie on the same corner line, because the dihedral angle of a unit tetrahedron is greater than $\pi / 6$. Therefore, $B, C, D$ lie in different corners, the equilateral triangle $B C D$ must be horizontal, and hence $\operatorname{size}(\mathbf{P})=1$. In this case, a small rotation around the line $B C$ makes $A, D$ interior vertices.

(2) Let $\Delta$ be the section of $\mathbf{P}$ by the $x y$-plane. We may suppose that none of $A, B, C, D$ is an interior vertex, and $\mathbf{P}$ has no empty face.

If $T$ has no corner vertex, then there is a face $\sigma$ of $\mathbf{P}$ that contains two vertices of $T$. Let $\ell$ be the line perpendicular to $\sigma$ and passing through the midpoint of the other two vertices. Then an appropriate rotation of $T$ around $\ell$ sends the two vertices not lying on $\sigma$ into $\mathbf{P}^{\circ}$.

Suppose that $T$ has only one corner vertex, say, $D$. Let $\sigma$ be the face opposite to $D$. Then one of $A, B, C$ does not lie on $\sigma$. To see this, suppose that $A, B, C$ lie on $\sigma$. Let $G$ be the barycenter of $A B C$. Then $D G$ is horizontal. Suppose that $\theta:=\angle G D \bar{A} \geq \angle G D \bar{B} \geq \angle G D \bar{C}$. Then $\theta$ attains its minimum when $\bar{C}$ is the midpoint of $\bar{A} \bar{B}$ (i.e., when $\bar{C}=G$ ). In this case, noting that $|D \bar{C}|=\sqrt{2 / 3}$ and $|\bar{A} \bar{C}|=1 / 2$, we have $\tan \theta=|\bar{A} \bar{C}| /|D \bar{C}|=\sqrt{3 / 8}>\sqrt{1 / 3}=\tan (\pi / 6)$, and thus $\theta>\pi / 6$. If $D$ is a corner vertex, then it follows from $\sigma \perp G D$ that $\theta \leq \pi / 6$, a contradiction. Thus $\sigma$ contains at most two of $A, B, C$.

If $\sigma$ contains two vertices of $T$, then a rotation of $T$ around the line through $D$ and perpendicular to $\sigma$ sends the remaining vertex into $\mathbf{P}^{\circ}$, and we are done. So, we may assume that $\sigma$ contains only one vertex of $T$, say, $C$. If $A, B$ lie on the same face, say $\tau$, then $A, B, D$ lie on $\tau$. Let $D X$ be a line segment obtained by cutting $\tau$ horizontally, and let $M$ be the midpoint of $D X$. Then a small rotation around the line through $M$ and perpendicular to $\tau$ sends $C$ into $\mathbf{P}^{\circ}$.

Thus, we may assume that $A, B$ lie on different faces. In this case, $A, B$ are both lower (or both higher) than $D$, for otherwise, $\angle A D B$ would be greater than $\pi / 3$. So, we may suppose that $z(A)<z(B)<z(D)$. Let $F$ be the midpoint of $A B$. Then $z(A)<z(F)<z(B)<z(D), \bar{A} \bar{D}<\bar{B} \bar{D}$ and $\angle \bar{D} \bar{F} \bar{A}<\angle \bar{D} \bar{F} \bar{B}$.

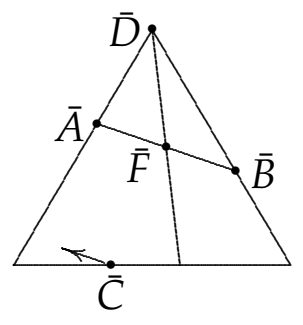

Figure 3: Just one corner vertex

To show that $\bar{A}$ and $\bar{C}$ lie in the same side of the line $\bar{D} \bar{F}$ in the $x y$-plane, suppose, on the contrary, that $\bar{B}$ and $\bar{C}$ lie on the same side. In this case, using 
$\angle \bar{D} \bar{F} \bar{B}>\pi / 2$ we have $\angle \bar{B} \bar{F} \bar{C}<\pi / 2$. Noting that $\angle B F C=\pi / 2$, we have $(z(B)>) z(F)>z(C)$. Similarly, using $\angle \bar{D} \bar{F} \bar{C}>\angle \bar{D} \bar{F} \bar{B}>\pi / 2$ and $\angle D F C<$ $\pi / 2$, we have $(z(D)>) z(F)<z(C)$, a contradiction. Thus, $\bar{A}$ and $\bar{C}$ must lie on the same side, see Figure 3.

Let us verify that $z(A)<z(C)$. If $\angle \bar{A} \bar{F} \bar{C}<\pi / 2$, then this follows from $\angle A F C=\pi / 2$ and $z(A)<z(F)$. Otherwise we have $\angle \bar{D} \bar{F} \bar{C}>\angle \bar{A} \bar{F} \bar{C}>\pi / 2$. Then $\angle D F C<\pi / 2$ and $z(D)>z(F)$ imply $z(F)<z(C)$, and thus $z(A)<$ $z(F)<z(C)$.

Thus, $z(A)<z(B), z(A)<z(C)$, and $A B \perp$ (the plane $D F C$ ). Now, if we rotate $T$ around the line $D F$ so that the inclination of $A B$ becomes steeper ( $B$ goes up, $A$ goes down in the $z$-direction), then $A$ and $B$ moves inward $\mathbf{P}$. In this case

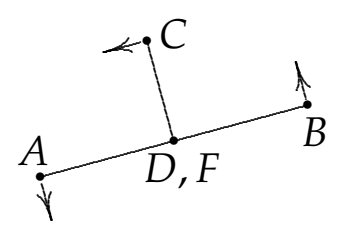

Figure 4: View in the direction from $F$ to $D$

the vertex $C$ moves in the direction $\overrightarrow{B A}$, see Figure 4 , and thus, $\bar{C}$ moves in the direction $\vec{B} \vec{A}$. Namely, $\bar{C}$ moves into the interior $\Delta^{\circ}$ of $\Delta$, because $|\bar{A} \bar{D}|<|\bar{B} \bar{D}|$, see Figure 3. Therefore, $C$ moves inward P. Hence all $A, B, C$ become interior points of $\mathbf{P}$.

\section{Minimal containment size of a unit tetrahedron}

Lemma 4.1. Let $T=A B C D \subset \mathbf{P}$ be an embedding such that $T$ has at least two corner vertices and has no interior vertex. Then the following holds.

(1) If $T$ is a tangential embedding, then size $(\mathbf{P})=t_{1}$, and the embedding is equivalent to one of $\beta(\sigma, \delta)$.

(2) If $T$ is not a tangential embedding, then size $(\mathbf{P})=t_{0}$ and the embedding is equivalent to one of $\alpha(\sigma, \epsilon)$.

Proof. Since two corner vertices cannot lie on the same corner line (because the dihedral angle of $T$ is greater than $\pi / 3), T$ cannot have three corner vertices, for otherwise, size $(\mathbf{P})$ would be 1 and one vertex would be an interior vertex. Hence $T$ has exactly two corner vertices.

(1) First suppose that $T$ is a tangential embedding. Let $A, B$ be the two corner vertices of $T$, and let $\sigma$ be the face of $\mathbf{P}$ that contains the edge $A B$. Then $C$ or $D$ lies on $\sigma$. This can be seen as follows: Suppose that none of $C, D$ lies on $\sigma$. Then, since $T$ is a tangential embedding, $C, D$ and one of $A, B$, say, $B$ lie on the 
same face of $\mathbf{P}$. Let $Z$ be the barycenter of $B C D$. We may suppose that $A Z$ lies on the $x y$-plane. Now, when we rotate $T$ around $A Z$, then the minimum value $\theta$ of $\max \{\angle Z A \bar{B}, \angle Z A \bar{C}, \angle Z A \bar{D}\}$ is attained in the case that one of $\bar{B}, \bar{C}, \bar{D}$, say $\bar{D}$ coincides with $Z$. In this case, since $|\bar{B} Z|=1 / 2$ and $|A Z|=\sqrt{2 / 3}$, we have $\tan \angle Z A \bar{B}=(1 / 2) / \sqrt{2 / 3}=\sqrt{3 / 8}>\sqrt{1 / 3}=\tan (\pi / 6)$. Therefore, $\theta>\pi / 6$. This implies that if $A$ is a corner vertex, and $B C D$ lie on the plane determined by the opposite face of the corner where $A$ is lying, then $A B C D$ is never contained in the prism $\mathbf{P}$. Thus, one of $C, D$, say $C$ lies on $\sigma$.

Let $G$ be the barycenter of $A B C$, and let $\tau$ be the face containing $D$. Then, $G D$ is horizontal. We may suppose that GD lie on the $x y$-plane. Suppose that $A \in \tau \cap \sigma$, see Figure 5 . Let us verify that this is $\beta(\sigma, \delta)$ for some $\delta$ given in the proof of Lemma 2.2, and size $\left(\mathbf{P}_{1}\right)=\mathbf{t}_{1}$.
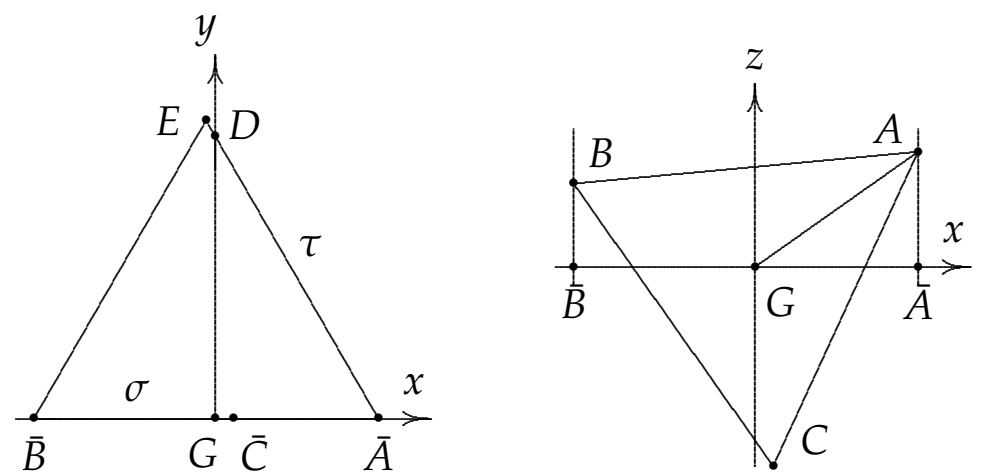

Figure 5: Top view of $\mathbf{P}_{1}$ and front view of $\sigma$

Let $\phi=\angle \bar{A} A G$. Then $\operatorname{size}\left(\mathbf{P}_{1}\right)=|\overline{\mathrm{A}} \overline{\mathrm{B}}|=|\mathrm{AB}| \sin (\angle \overline{\mathrm{A}} \mathrm{AB})=\sin (\angle \overline{\mathrm{A}} \mathrm{AG}+$ $\angle \mathrm{GAB})=\sin (\phi+\pi / 6)$. On the other hand, using $|G D|=\sqrt{2 / 3}$ and $\angle \bar{A} D G=$ $\pi / 6$, we have $|\bar{A} G|=\sqrt{2} / 3$, and thus $\sin \phi=|\bar{A} G| /|A G|=\sqrt{2 / 3}, \cos \phi=$ $1 / \sqrt{3}$. Hence

$$
\bar{A} \bar{B}=\sin (\phi+\pi / 6)=\sin \phi \cos (\pi / 6)+\cos \phi \sin (\pi / 6)=(3 \sqrt{2}+\sqrt{3}) / 6,
$$

namely, $\operatorname{size}\left(\mathbf{P}_{1}\right)=|\overline{\mathrm{A}} \overline{\mathrm{B}}|=\mathrm{t}_{1}$, which proves the tangential embedding case.

(2) Now we consider the non-tangential embedding case. Let $B, C$ be the two corner vertices of $T$. Then, none of $A, D$ lies on the face of $\mathbf{P}$ that conains the edge $B C$, and $A, D$ lie on different faces of $\mathbf{P}$.

Let us show that $\bar{A} \bar{D} \| \bar{B} \bar{C}$. Let $\sigma$ be the face of $\mathbf{P}$ that contains $B C$. Let $\Pi$ be the plane that perpendicularly bisects $B C$. Then $A, D$ lie on $\Pi$. Let $X Y Z$ be the section of $\mathbf{P}$ by $\Pi, Y Z$ be the line segment $\Pi \cap \sigma, X$ be the intersection point of $\Pi$ and the corner line of $\mathbf{P}$ opposite to $\sigma$. Let $M$ be the midpoint of $B C$ (and hence the midpoint of $Y Z$ ). Then the segment $X M$ is horizontal. Thus $X Y Z$ is an isosceles triangle with base $Y Z$, and $A, D$ lie on $X Y \cup X Z$. Since $|M X|<\sqrt{3} / 2$ 
and $|B M|=|C M|=1 / 2$, the locus $\gamma$ of points on $\Pi$ that are at unit distance apart from $B$ (and $C$ ) is a circle with center $M$, radius $\sqrt{3} / 2$. Since $X$ lies inside the circle $\gamma, X Y$ intersects $\gamma$ at a single point, and also $X Z$ intersects $\gamma$ at a single point. Thus $(X Y \cup X Z) \cap \gamma$ consists of two points, and they must be $A$ and $D$, see Figure 6. Since $X Y Z$ is an isosceles triangle with base $Y Z$, we have $A D \| Y Z$, and hence $\bar{A} \bar{D} \| \bar{Y} \bar{Z}$. Since the two lines $\bar{B} \bar{C}$ and $\bar{Y} \bar{Z}$ are the same line, we have $\bar{A} \bar{D} \| \bar{B} \bar{C}$.

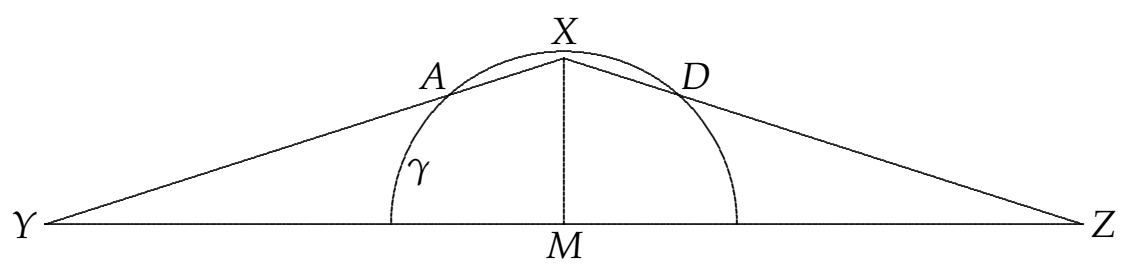

Figure 6: On the plane $\Pi$ that bisects $B C$ perpendicularly

Thus, $\bar{A} \bar{B} \bar{C} \bar{D}$ (or $\bar{D} \bar{B} \bar{C} \bar{A}$ ) is a trapezoid in $\Delta$ with all vertices on $\partial \Delta$, and $\bar{B} \bar{C}$ is an edge of $\Delta$, just as shown in Figure 1. Let us find the edge length $t=|\bar{B} \bar{C}|$ of $\triangle$. Since the height of the trapezoid is the distance between the opposite edges of $A B C D$, it is equal to $1 / \sqrt{2}$. Then, by comparing the heights of the equilateral triangles $\bar{A} \bar{D} X$ and $\bar{B} \bar{C} X$, we have $|\bar{A} \bar{D}|: t=(\sqrt{3} t / 2-1 / \sqrt{2}): \sqrt{3} t / 2$, and thus $|\bar{A} \bar{D}|=t-\sqrt{2 / 3}$. Let $\theta$ be the angle of inclination of $A D$. Then, since $\bar{A} \bar{D} \| \bar{B} \bar{C}$, the angle of inclination of $B C$ is $\pi / 2-\theta$. Hence $t-\sqrt{2 / 3}=|\bar{A} \bar{D}|=\cos \theta$ and $t=|\bar{B} \bar{C}|=\sin \theta$. Therefore, $1=(t-\sqrt{2 / 3})^{2}+t^{2}$, and solving this equation we have $t=(1+\sqrt{2}) / \sqrt{6}$. This proves that $\operatorname{size}(\mathbf{P})=t_{0}$ and $T$ is equivalent to one of $\alpha(\sigma, \epsilon)$.

Lemma 4.2. For any embedding $T \subset \mathbf{P}(t)$, there is the minimum value $s_{0}$ such that $T$ is equivalent to $T_{0} \subset \mathbf{P}\left(s_{0}\right) \subset \mathbf{P}(t)$ in $\mathbf{P}(t)$. Moreover, $s_{0}=t_{0}$ or $s_{0}=t_{1}$.

Proof. Let $s_{0}=\inf \left\{s \leq t \mid \exists T^{\prime} \subset \mathbf{P}(s)\right.$ such that $T \sim T^{\prime}$ in $\left.\mathbf{P}(t)\right\}$. Then there is a sequence of points $\left(A_{n}, B_{n}, C_{n}, D_{n}\right) \in \mathbb{R}^{12}, n=1,2,3, \ldots$, and a sequence $s_{n} \in \mathbb{R}_{+}, n=1,2,3, \ldots$, such that for each $n$,

1. $T_{n}:=A_{n} B_{n} C_{n} D_{n}$ is a unit tetrahedron contained in $\mathbf{P}\left(s_{n}\right) \cap[-2 \leq z \leq 2]$,

2. $T \sim T_{n}$ in $\mathbf{P}(t)$, and

3. $\lim s_{n}=s_{0}$,

where $[-2 \leq z \leq 2]:=\left\{(x, y, z) \in \mathbb{R}^{3} \mid-2 \leq z \leq 2\right\}$. Since $\mathbf{P}(t) \cap[-2 \leq z \leq 2]$ is compact, a convergent subsequence $\left(A_{m}, B_{m}, C_{m}, D_{m}\right)$ exists and converges to $\left(A_{0}, B_{0}, C_{0}, D_{0}\right)$. Then $T_{0}:=A_{0} B_{0} C_{0} D_{0}$ is a unit tetrahedron contained in $\mathbf{P}\left(s_{0}\right)$. Let $c_{m}=1 / 2^{2}+1 / 2^{3}+\cdots+1 / 2^{m}$. Since $T_{m} \sim T_{m+1}$ in $\mathbf{P}(t)$, there is a motion $F_{m}: T_{m} \times\left[c_{m}, c_{m+1}\right] \rightarrow \mathbf{P}(t)$ of $T_{m}$ that sends $T_{m}$ to $T_{m+1}$ and a motion $F: T \times$ 
$[0,1 / 2] \rightarrow \mathbf{P}(t)$ that send $T$ to $T_{1}$. Connecting these motions, we have a motion $F: T \times[0,1) \rightarrow \mathbf{P}(t)$. This motion can be extended to $F: T \times[0,1] \rightarrow \mathbf{P}(t)$ by putting $F(A, 1)=A_{0}, \ldots, F(D, 1)=D_{0}$ and extending linearly for all $x \in T$. Then $F$ is a continuous map and a motion of $T$ to $T_{0}$. Since $s_{0}$ is the minimum containment size, $T_{0}$ satisfies neither (1) nor (2) of Lemma 3.2. Hence, by Lemma 4.1 , we have $s_{0}=t_{0}$ or $s_{0}=t_{1}$.

Corollary 4.1. For $t_{0} \leq s<t_{1}$, every embedding $T \subset \mathbf{P}(s)$ is equivalent to one of $\alpha(\sigma, \epsilon)$, and for $t_{1} \leq t$, every embedding $T \subset \mathbf{P}(t)$ is equivalent to one of $\alpha(\sigma, \epsilon)$ or one of $\beta(\sigma, \delta)$.

\section{Territories and borders in a prism}

In a prism $\mathbf{P}$, the territory of a corner of $\mathbf{P}$ consists of those points of $\mathbf{P}$ that are nearer to the corner than to other corners. Each territory is a quadrilateral prism, and the three territories are mutually congruent. A border is the intersection of any two territories, see Figure 7.

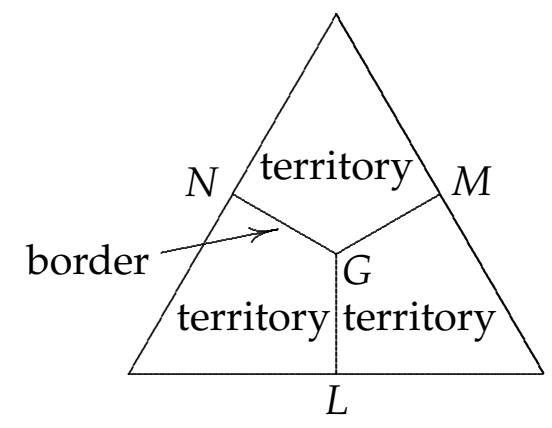

Figure 7: Territories and borders of $\mathbf{P}$, top view

Lemma 5.1. Let $\mathbf{P}$ be a prism of size $t<1$ and let $T \subset \mathbf{P}$ be a unit tetrahedron. Then no vertex of $T$ lies on a border.

Proof. Let $T=A B C D$ and suppose that $A$ lies on a border. We may assume that $z(A)=0<z(B)$. Let $\Delta$ be the section of $\mathbf{P}$ by the plane $z=0, G$ be the barycenter of $\Delta$, and $L, M, N$ be the midpoints of the edges of $\Delta$, see Figure 7 . Then $A$ lies on $G L \cup G M \cup G N$. We may suppose that $A$ lies on $G M$. Let $\Omega$ be the intersection of $\mathbf{P}$ and the unit sphere with center $A$. This intersection $\Omega$ is the union of two connected surfaces, $\Omega^{+}$in the half space $z>0$ and $\Omega^{-}$in the half space $z<0$. Figure 8 shows the upper surface $\Omega^{+}$. The vertex $B$ lies on $\Omega^{+}$. Let $P, Q, R$ be the corner point such that $|A P|=|A Q|=|A R|=1$ and $z(P)=z(Q)>z(R)>0$. Then $\Omega^{+}$intersects the faces of $\mathbf{P}$ in three circular arcs $\widehat{P Q}, \widehat{Q R}, \widehat{R P}$. Let $S$ be the corner point on the same corner line as $R$ such that $z(S)=z(P)$. (If $A=G$, then $R$ 
coincides with $S$.) Let the arcs $\widehat{R Q}$ and $\widehat{R P}$ cross $S Q$ and $S P$ at $U, V$, respectively. If $A=G$ then $R=S=U=V$, and if $A=M$, then $U$ is the midpoint of $S Q$ and $z(R)=\sqrt{1-3 t^{2} / 4}$. Hence we have

$$
|S U|=|S V| \leq t / 2, \quad 1 / 2<\sqrt{1-3 t^{2} / 4} \leq z(R) \leq z(P) \leq \sqrt{1-t^{2} / 4}<1 .
$$

Thus, $\Omega^{+}$is contained in the open half space $z>1 / 2$. Similarly, $\Omega^{-}$is contained in the open half space $z<-1 / 2$. Since $B$ lies on $\Omega^{+}$, the remaining vertices $C, D$ must also lie on $\Omega^{+}$.

From (1), we have $|R U|=|R V|<1,|U V|<1,|P Q|=|Q S|=|S P|<1$. Hence, we can deduce that

$$
\max \{\angle P A Q, \angle P A S, \angle Q A S, \angle U A V, \angle U A R, \angle V A R\}<\pi / 3 .
$$

Now, we divide $\Omega^{+}$by the plane $z=z(P)$ into two surfaces; $\Omega_{1}^{+}$, the upper part, and $\Omega_{2}^{+}$, the lower part. Here, we note that if $X, Y$ belong the tetrahedron $A P Q S$, then $\angle X A Y \leq \max \{\angle P A Q, \angle P A S, \angle Q A S\}$. (Proof of this fact will be elementary.) From this fact it follows that for any points $X, Y \in \Omega_{1}^{+}, \angle X A Y<\pi / 3$. This implies that the diameter of $\Omega_{1}^{+}$is less than 1 , and hence $\Omega_{1}^{+}$cannot contain more than one vertex of $T$. Similarly, the diameter of $\Omega_{2}^{+}$is less than 1 , and it cannot contain more than one vertex of $T$. Therefore, $\Omega^{+}$cannot contain the three vertices $B, C, D$, which is a contradiction.

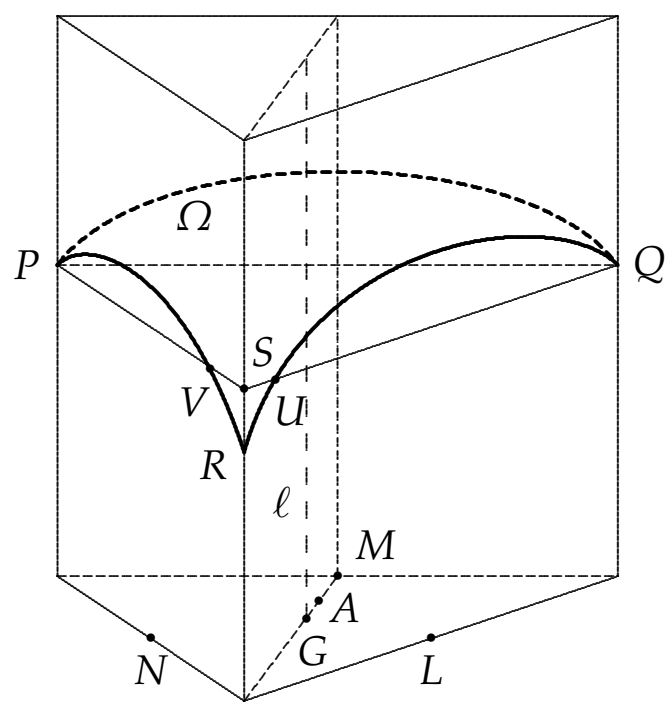

Figure 8: A section by the unit sphere with center $A$

Let $T \subset \mathbf{P}$ be an embedding into a prism of size $t<1$. Then, since $\mathbf{P}$ cannot contain a horizontal line segment of length 1 , we can label the vertices of $T$ with 
$A_{1}, A_{2}, A_{3}, A_{4}$ so that $z\left(A_{1}\right)<z\left(A_{2}\right)<z\left(A_{3}\right)<z\left(A_{4}\right)$. We call the vertex of label $A_{i}$ the $i$ th vertex of $T$. Notice that the labels of the vertices of $T$ do not vary under any continuous motion of $T$ within $\mathbf{P}$.

Lemma 5.2. Let $T_{1}, T_{2} \subset \mathbf{P}(t)\left(t_{0} \leq t<1\right)$ be two embeddings of a unit tetrahedron. If, for some $i=1,2,3,4$, the ith vertex of $T_{1}$ and the ith vertex of $T_{2}$ lie in different territories, then $T_{1}$ and $T_{2}$ are not equivalent.

Proof. If $T_{1}$ and $T_{2}$ are equivalent then there is a motion of $T_{1}$ in $\mathbf{P}(t)$ which sends the $i$ th vertex of $T_{1}$ to the $i$ th vertex of $T_{2}$. Since they belong different territories in the begining, the $i$ th vertex of $T_{1}$ must cross a border in the midway, which is impossible by Lemma 5.1 .

Lemma 5.3. If $T \subset \mathbf{P}$, size $(\mathbf{P})=t<1$, then each territory of $\mathbf{P}$ contains a vertex of $T$.

Proof. Since the width of the union of two territories is $\left(\frac{\sqrt{3}}{4}\right) t$ (see Figure 7 ) which is smaller than $1 / \sqrt{2}$, the width of $T$ (see [10] or [6]), the convex hull of two territories cannot contain $T$. Hence each territory contains a vertex of $T$.

Lemma 5.4. Let $\mathbf{P}$ be a prism of size $t<1$ and $T \subset \mathbf{P}$ be a unit tetrahedron. Suppose that the vertices $A_{1}, A_{4}$ of $T$ lie in the territory of a corner line $\ell$. Then the line $A_{1} A_{4}$ is never parallel to (or never contained in) the plane that bisects the dihedral angle at $\ell$.

Proof. Suppose that $A_{1} A_{4}$ is parallel to the plane $H$ that bisects the dihedral angle at $\ell$. We may suppose that $H$ is the $x z$-plane in $\mathbb{R}^{3}$. Let $K$ be the plane that perpendicularly bisects the edge $A_{1} A_{4}$. Then, $K$ intersects $H$ orthogonally. Hence the section of $\mathbf{P}$ by $K$ is an isosceles triangle $X Y Z$ with base $Y Z$ in the face of $\mathbf{P}$ opposite to $\ell$. Then $|Y Z|=t$ and $s:=|X Y|=|X Z|>t$. Let $L, M, N$ be the midpoints of $Y Z, Z X, X Y$, respectively, and let $G$ be the barycenter of $X Y Z$ as shown in Figure 9. Since the width of a unit tetrahedron is $1 / \sqrt{2}$, we have $z\left(A_{4}\right)-z\left(A_{1}\right) \geq 1 / \sqrt{2}$. Hence the angle between the line $A_{1} A_{4}$ and the $x y$-plane is at least $\pi / 4$, and hence the angle between $K$ and the $x y$-plane is at most $\pi / 4$. Therefore, $|X L|$ is at most $\sqrt{2} \times\left(\frac{\sqrt{3}}{2}\right) t$, and hence $s=|X Z|=|X Y| \leq\left(\frac{\sqrt{7}}{2}\right) t$.

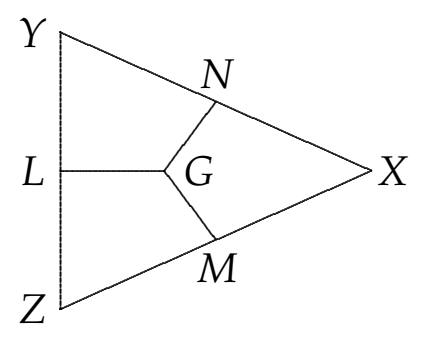

Figure 9: Section of $\mathbf{P}$ by the perpendicular bisector of $A D$

Since $A_{2}, A_{3}$ lie on the plane $K$, and hence lie on the isosceles triangle $X Y Z$. Since $A_{1}, A_{4}$ are in the territory containing $X$, the vertices $A_{2}, A_{3}$ must lie in the 
pentagon YZMGN. On the other hand, by applying the parallelogram theorem, we have

$$
\begin{aligned}
|Y M|^{2}=|Z N|^{2} & =\frac{1}{2}|Z Y|^{2}+\frac{1}{2}|Z X|^{2}-|X N|^{2} \\
& =\frac{1}{2} t^{2}+\frac{1}{2} s^{2}-\frac{1}{4} s^{2}=\frac{1}{2} t^{2}+\frac{1}{4} s^{2} \\
& \leq \frac{1}{2} t^{2}+\frac{1}{4}\left(\frac{\sqrt{7}}{2} t\right)^{2}=\frac{15}{16} t^{2}<1 .
\end{aligned}
$$

Hence the diamter of the pentagon $Y Z M G N$ is less than 1 . This implies that the pentagon $Y Z M G N$ cannot contain $\left\{A_{2}, A_{3}\right\}$, a contradiction.

\section{Proof of the theorem}

Lemma 6.1. If $t_{0} \leq t<1$, the six $\alpha=\alpha(\sigma, \epsilon)$ are mutually non-equivalent in $\mathbf{P}(t)$.

Proof. Let $\sigma_{1}, \sigma_{2}, \sigma_{3}$ be the faces of $\mathbf{P}(t)$ such that $A_{3}$ of $\alpha\left(\sigma_{1}, \epsilon_{1}\right)$ (the cross embedding constructed in the proof of Lemma 2.1) lies on the line $\sigma_{1} \cap \sigma_{2}$. We prove that $\alpha\left(\sigma_{i}, \epsilon_{j}\right), i=1,2,3, j=1,2$ are all non-equivalent in $\mathbf{P}(t)$. Proof is given by the following table. Let us explain what means a number in a cell of the table. Look at, for instance, the cell in the low of $\alpha\left(\sigma_{2}, \epsilon_{2}\right)$ and the column of $\alpha\left(\sigma_{3}, \epsilon_{1}\right)$. The number in this cell is 1 . This means that the first vertex of $\alpha\left(\sigma_{2}, \epsilon_{2}\right)$ and the first vertex of $\alpha\left(\sigma_{3}, \epsilon_{1}\right)$ lie in different territories. Then by Lemma 5.2, we have $\alpha\left(\sigma_{2}, \epsilon_{2}\right) \nsim \alpha\left(\sigma_{3}, \epsilon_{1}\right)$ in $\mathbf{P}(t)$. Now it is easy to check that the entries in the cells are all correct.

\begin{tabular}{|c||c|c|c|c|c|c|}
\hline & $\alpha\left(\sigma_{1}, \epsilon_{1}\right)$ & $\alpha\left(\sigma_{1}, \epsilon_{2}\right)$ & $\alpha\left(\sigma_{2}, \epsilon_{1}\right)$ & $\alpha\left(\sigma_{2}, \epsilon_{2}\right)$ & $\alpha\left(\sigma_{3}, \epsilon_{1}\right)$ & $\alpha\left(\sigma_{3}, \epsilon_{2}\right)$ \\
\hline$\alpha\left(\sigma_{1}, \epsilon_{1}\right)$ & - & 2 & 1 & 1 & 1 & 1 \\
\hline$\alpha\left(\sigma_{1}, \epsilon_{2}\right)$ & 2 & - & 1 & 1 & 1 & 1 \\
\hline$\alpha\left(\sigma_{2}, \epsilon_{1}\right)$ & 1 & 1 & - & 2 & 1 & 1 \\
\hline$\alpha\left(\sigma_{2}, \epsilon_{2}\right)$ & 1 & 1 & 2 & - & 1 & 1 \\
\hline$\alpha\left(\sigma_{3}, \epsilon_{1}\right)$ & 1 & 1 & 1 & 1 & - & 2 \\
\hline$\alpha\left(\sigma_{3}, \epsilon_{2}\right)$ & 1 & 1 & 1 & 1 & 2 & - \\
\hline
\end{tabular}

Lemma 6.2. For $t_{1} \leq t<1$, the twelve $\beta(\sigma, \delta)$ are mutually non-equivalent in $\mathbf{P}(t)$.

Proof. First we show that $\beta\left(\sigma, \delta_{i}\right), i=1,2,3,4$ are all non-equivalent in $\mathbf{P}(t)$. Proof is given by the following table.

\begin{tabular}{|c||c|c|c|c|}
\hline- & $\beta\left(\sigma, \delta_{1}\right)$ & $\beta\left(\sigma, \delta_{2}\right)$ & $\beta\left(\sigma, \delta_{3}\right)$ & $\beta\left(\sigma, \delta_{4}\right)$ \\
\hline$\beta\left(\sigma, \delta_{1}\right)$ & - & 1 & 2 & 1 \\
\hline$\beta\left(\sigma, \delta_{2}\right)$ & 1 & - & 1 & 2 \\
\hline$\beta\left(\sigma, \delta_{3}\right)$ & 2 & 1 & - & 1 \\
\hline$\beta\left(\sigma, \delta_{4}\right)$ & 1 & 2 & 1 & - \\
\hline
\end{tabular}


Now it will be sufficient to show that if $\sigma_{1} \neq \sigma_{2}$, then $\beta\left(\sigma_{1}, \delta_{i}\right)$ and $\beta\left(\sigma_{2}, \delta_{j}\right)$ are not equivalent in $\mathbf{P}(t)$ for all $i, j$. To make the argument clear, we may suppose that $\sigma_{1} \cap \sigma_{2}$ contains the vertex $A_{4}$ (the highest vertex) of $\beta\left(\sigma_{1}, \delta_{1}\right)$. Then, we have the following incomplete table with two blank cells.

\begin{tabular}{|c||c|c|c|c|}
\hline- & $\beta\left(\sigma_{2}, \delta_{1}\right)$ & $\beta\left(\sigma_{2}, \delta_{2}\right)$ & $\beta\left(\sigma_{2}, \delta_{3}\right)$ & $\beta\left(\sigma_{2}, \delta_{4}\right)$ \\
\hline$\beta\left(\sigma_{1}, \delta_{1}\right)$ & 1 & 2 & 1 & \\
\hline$\beta\left(\sigma_{1}, \delta_{2}\right)$ & 1 & 1 & 1 & 1 \\
\hline$\beta\left(\sigma_{1}, \delta_{3}\right)$ & 1 & & 1 & 2 \\
\hline$\beta\left(\sigma_{1}, \delta_{4}\right)$ & 1 & 1 & 1 & 1 \\
\hline
\end{tabular}

Let us show that $\beta\left(\sigma_{1}, \delta_{1}\right)$ and $\beta\left(\sigma_{2}, \delta_{4}\right)$ (corresponding to the upper-right blank cell) are not equivalent in $\mathbf{P}(t)$.

Note that in both $\beta\left(\sigma_{1}, \delta_{1}\right)$ and $\beta\left(\sigma_{2}, \delta_{4}\right)$, the vertices $A_{1}, A_{4}$ lie in the territory of the corner line $\ell:=\sigma_{1} \cap \sigma_{2}$. Let $H$ be the plane that bisects the dihedral angle at the corner $\ell$ of $\mathbf{P}(t)$. Let $d(P, H)$ denote the distance from a point $P$ to the plane $H$. Then, in $\beta\left(\sigma_{1}, \delta_{1}\right)$, we have $d\left(A_{1}, H\right)>0, d\left(A_{4}, H\right)=0$, whereas, in $\beta\left(\sigma_{2}, \delta_{4}\right)$, we have $d\left(A_{1}, H\right)=0, d\left(A_{4}, H\right)>0$. Therefore, if $\beta\left(\sigma_{1}, \delta_{1}\right) \sim \beta\left(\sigma_{2}, \delta_{4}\right)$, then on the way of the motion of $\beta\left(\sigma_{1}, \delta_{1}\right)$ from its original position to the position of $\beta\left(\sigma_{2}, \delta_{4}\right)$, there must be a moment $d\left(A_{1}, H\right)=d\left(A_{4}, H\right)$ holds. But this is impossible by Lemma 5.4. Hence $\beta\left(\sigma_{1}, \delta_{1}\right) \nsim \beta\left(\sigma_{2}, \delta_{4}\right)$ in $\mathbf{P}(t)$. Similarly, it can be proved by applying Lemma 5.4 that $\beta\left(\sigma_{1}, \delta_{3}\right)$ and $\beta\left(\sigma_{2}, \delta_{2}\right)$ (the ones corresponding to the other blank cell) are not equivalent in $\mathbf{P}(t)$. Thus, all twelve $\beta(\sigma, \delta)$ are mutually non-equivalent in $\mathbf{P}(t)$.

Corollary 6.1. Let $t_{1} \leq t<1$. Then no $\beta$ is equivalent to an $\alpha$ in $\mathbf{P}(t)$.

Proof. If some $\beta$ is equivalent to some $\alpha$ in $\mathbf{P}(t)$, then every $\beta$ would be equivalent to an $\alpha$ in $\mathbf{P}(t)$. However, mutually non-equivalent twelve $\beta$ s cannot be equivalent to $\operatorname{six} \alpha \mathrm{s}$.

\section{Proof of Theorem 1.1.}

By Lemma 4.2, $v(t)=0$ for $t<t_{0}$, and by Corollary 4.1 and Lemma 6.1, we have $v(t)=6$ for $t_{0} \leq t<t_{1}$. By Corollary 4.1, Lemmas 6.1, 6.2 and Corollary 6.1 , it follows that $v(t)=6+12=18$ for $t_{1} \leq t<1$.

Now, suppose that $t=1$. Then, every $T \subset \mathbf{P}(1)$ is equivalent to some $\alpha$ or some $\beta$ by Corollary 4.1. Suppose that $T \subset \mathbf{P}(1)$ is equivalent to some $\alpha$, say, to the cross embedding $A B C D \subset \mathbf{P}\left(t_{0}\right)$ given in the proof of Lemma 2.1. Then by applying a translation along the $y$-axis, we may suppose that the edge $B C$ lies on a face, say $\sigma$ of $\mathbf{P}(1)$, and the line $L$ passing through the midpoint of $B C$ and perpendicular to $\sigma$ meets the corner line opposite to the face $\sigma$. Then, by rotating around the line $L$, we can move $A B C D$ within $\mathbf{P}(1)$ so that $B C$ becomes horizontal and $A B$ becomes vertical. Now, rotating the resulting tetrahedron around the horizontal line $B C$ within $\mathbf{P}(1)$ so that a face of the tetrahedron becomes horizontal, and one vertex lies above the horizontal face. 
Next, suppose that $T \subset \mathbf{P}(1)$ is equivalent to a $\beta$, say, to the tangential embedding $A B C D \subset \mathbf{P}\left(t_{1}\right)$ given in the proof of Lemma 2.2. We can translate $A B C D$ so that $A$ comes to the corner line and $A B C$ lie on a face $\sigma$ of $\mathbf{P}(1)$. Now, by rotating $A B C D$ around the line passing through $A$ and perpendicular to the face $\sigma$, we can make the line $A B$ horizontal. Then, rotate around the edge $A B$, we can make one face of the tetrahedron horizontal, and one vertex lies above the face. Thus, every embedding $T \subset \mathbf{P}(1)$ is equivalent to an embedding in which one face is horizontal and one vertex is above the horizontal face. Therefore $v(1)=1$, and hence $v(t)=1$ for $t \geq 1$. This completes the proof.

\section{References}

[1] I. Bárány, H. Maehara, N. Tokushige, Tetrahedra passing through a triangular hole, submitted.

[2] R. Brandenberg, T. Theobald, Radii minimal projections of polytopes and constrained optimization of symmetric polynomials, Adv. Geom. 6(2006) 7183.

[3] R. Brandenberg, T. Theobald, Algebraic method for computing smallest enclosing and circumscribing cylinders of simplices, Appl. Algebra Engrg. Comm. Comput. 14(2004) 439-460.

[4] O. Devillers, B. Mourrain, F. P. Preparata, P. Trebuchet, Circular cylinders through four or five points in space, Discrete Comput. Geom. 29(2003) 83-104.

[5] J. Itoh, Y. Tanoue, T. Zamfirescu, Tetrahedra passing through a circular or square hole, Rend. Circ. Mat. Palermo (2) Suppl. No. 77 (2006) 349-354.

[6] H. Maehara, An extremal problem for arrangements of great circles, Math. Japonica 41, No. 1 (1995) 125-129.

[7] H. Maehara, On congruent embeddings of a tetrehedron into a circular cylinder, preprint.

[8] V. Pukhov, Kolmogorov diameter of a regular simplex, Mosc. Univ. Math. Bull. 35(1980) 38-41.

[9] E. Schömer, J. Sellen, M. Reichmann, C. Yap, Smallest enclosing cylinders, Algorithmica 27(2000) 170-186.

[10] P. Steinhagen, Über die grösste Kugel in einer konvexen Punktmenge, Abh. Math. Sem. Hamburg 1(1921) 15-26. 